\title{
Starlikeness of Functions Defined by Third-Order Differential Inequalities and Integral Operators
}

\author{
R. Chandrashekar, ${ }^{1}$ Rosihan M. Ali, ${ }^{2}$ K. G. Subramanian, ${ }^{3}$ and A. Swaminathan ${ }^{4}$ \\ ${ }^{1}$ Department of Technology Management, Faculty of Technology Management and Business, \\ Universiti Tun Hussein Onn Malaysia, 86400 Parit Raja, Batu Pahat, Johor, Malaysia \\ ${ }^{2}$ School of Mathematical Sciences, Universiti Sains Malaysia, 11800 Penang, Malaysia \\ ${ }^{3}$ School of Computer Sciences, Universiti Sains Malaysia, 11800 Penang, Malaysia \\ ${ }^{4}$ Department of Mathematics, I.I.T. Roorkee, Roorkee 247667, India
}

Correspondence should be addressed to Rosihan M. Ali; rosihan@cs.usm.my

Received 3 January 2014; Accepted 12 March 2014; Published 3 April 2014

Academic Editor: Om P. Ahuja

Copyright (C) 2014 R. Chandrashekar et al. This is an open access article distributed under the Creative Commons Attribution License, which permits unrestricted use, distribution, and reproduction in any medium, provided the original work is properly cited.

Sufficient conditions are obtained to ensure starlikeness of positive order for analytic functions defined in the open unit disk satisfying certain third-order differential inequalities. As a consequence, conditions for starlikeness of functions defined by integral operators are obtained. Connections are also made to earlier known results.

\section{Introduction}

Let $\mathscr{H}$ denote the class of analytic functions $f$ defined in the open unit disk $U:=\{z \in \mathbb{C}:|z|<1\}$. For $a \in \mathbb{C}$ and $n$ a positive integer, let

$$
\mathscr{H}_{n}(a)=\left\{f \in \mathscr{H}: f(z)=a+\sum_{k=n}^{\infty} a_{k} z^{k}\right\},
$$

and $\mathscr{A}_{n}=\left\{f \in \mathscr{H}: f(z)=z+\sum_{k=n+1}^{\infty} a_{k} z^{k}\right\}$, with $\mathscr{A}_{1}:=\mathscr{A}$. For $\beta \in[0,1)$, denote by $\mathcal{S}^{*}(\beta)$ the subclass of $\mathscr{A}$ consisting of functions starlike of order $\beta$ satisfying

$$
\operatorname{Re}\left(\frac{z f^{\prime}(z)}{f(z)}\right)>\beta, \quad z \in U \text {. }
$$

The class $\mathcal{S}^{*}:=\mathcal{S}^{*}(0)$ is the well-known subclass of starlike functions studied widely in geometric function theory.

In the sequel, we give emphasis to the class

$$
\mathcal{S}_{1}(\beta)=\left\{f \in \mathscr{A}:\left|\frac{z f^{\prime}(z)}{f(z)}-1\right|<1-\beta, z \in U\right\},
$$

$\beta \in[0,1)$, where $\mathcal{S}_{1}:=\mathcal{S}_{1}(0) \subset \mathcal{S}^{*}$. Evidently $\mathcal{S}_{1}(\beta) \subset$ $\mathcal{S}^{*}(\beta)$ for $0 \leq \beta<1$. The class $\mathcal{S}_{1}(\beta)$ was investigated by
Silverman [1], who showed that $\mathcal{S}_{1}(\beta)$ coincides with $\mathcal{\delta}^{*}(\beta)$ for univalent functions with negative coefficients. This class has subsequently been studied in several other works (see, e.g., $[2])$.

The problem of determining sufficient conditions to ensure starlikeness of functions has been widely investigated. These include conditions in terms of differential inequalities; see, for example, [2-11]. Miller and Mocanu [12], Kuroki and Owa [13], and, more recently, Ali et al. [14] determined conditions for starlikeness of functions defined by an integral operator of the form

$$
f(z)=\int_{0}^{1} W(r, z) d r,
$$

or by the double integral operator

$$
f(z)=\iint_{0}^{1} W(r, s, z) d r d s .
$$

In this paper, conditions on certain third-order differential inequalities are found that would imply starlikeness of positive order. As a consequence, conditions on the kernel of certain integral operators are also obtained to ensure that the 
functions defined by these operators are starlike. Connections are also made to earlier known results.

Recall that an analytic function $f$ is subordinate to an analytic function $g$ in $U$, written as $f(z) \prec g(z)$, if there exists an analytic self-map $w$ of $U$ with $w(0)=0$ satisfying $f(z)=g(w(z))$.

The following lemmas will be required in the sequel.

Lemma 1 (see [15, Theorem 1, page 192] and see also [16, Theorem 3.1b, page 71]). Let $h$ be convex in $U$ with $h(0)=a$, $\gamma \neq 0$ and $\operatorname{Re} \gamma \geq 0$. If $p \in \mathscr{H}_{n}(a)$ and

$$
p(z)+\frac{z p^{\prime}(z)}{\gamma} \prec h(z),
$$

then

$$
p(z) \prec q(z) \prec h(z)
$$

where

$$
q(z)=\frac{\gamma}{n z^{\gamma / n}} \int_{0}^{z} h(t) t^{(\gamma / n)-1} d t
$$

The function $q$ is convex and is the best $(a, n)$-dominant.

Lemma 2 (see [17] and see also [16, Theorem 3.1d, page 76]). Let $h$ be a starlike function with $h(0)=0$. If $p \in \mathscr{H}_{n}(a)$ satisfies

$$
z p^{\prime}(z) \prec h(z)
$$

then

$$
p(z) \prec q(z)=a+\frac{1}{n} \int_{0}^{z} \frac{h(t)}{t} d t .
$$

The function $q$ is convex and is the best $(a, n)$-dominant.

\section{Main Results}

The following two results are easily obtained by simple adaptations of Theorem 2.1 and Theorem 2.6 in [13]. The proofs are therefore omitted.

Lemma 3. Let $f \in \mathscr{A}_{n}, 0 \leq \alpha<n \delta$, and $0 \leq \beta<1$. If

$$
\left|\delta z f^{\prime \prime}(z)-\alpha\left(f^{\prime}(z)-1\right)\right|<\frac{(n+1)(1-\beta)(n \delta-\alpha)}{n+1-\beta},
$$

then $f \in \mathcal{S}^{*}(\beta)$ with an extremal function $f(z)=z+(1-$ $\beta) z^{n+1} /(n+1-\beta)$.

Lemma 4. Let $0 \leq \alpha<n \delta, 0 \leq \beta<1$, and $g \in \mathscr{H}$. If

$$
|g(z)|<\frac{(n+1)(1-\beta)(n \delta-\alpha)}{n+1-\beta},
$$

then

$$
f(z)=z+\frac{z^{n+1}}{\delta} \iint_{0}^{1} g(r s z) r^{[(n-1) \delta-\alpha] / \delta} s^{n} d r d s
$$

is a starlike function of order $\beta$.
Remark 5. Even though the conditions given in Lemmas 3 and 4 are sufficient to deduce $f \in \mathcal{S}^{*}(\beta)$, they are in fact sufficient to imply $f \in \mathcal{S}_{1}(\beta) \subset \mathcal{S}^{*}(\beta)$.

The above two lemmas are next used to obtain conditions in terms of a third-order differential inequality and a thirdorder integral operator to deduce starlikeness of $f$ of order $\beta$.

Theorem 6. Let $f \in \mathscr{A}_{n}, 0<\alpha<n v, \delta>\alpha \geq \gamma \geq 0$, and $0 \leq \beta<1$. Further let $\mu$ and $\nu$ satisfy

$$
\nu-\alpha \mu=\delta-\gamma, \quad \nu \mu=\gamma
$$

If

$$
\begin{gathered}
\left|\gamma z^{2} f^{\prime \prime \prime}(z)+\delta z f^{\prime \prime}(z)-\alpha\left(f^{\prime}(z)-1\right)\right| \\
<\frac{(1+n \mu)(n+1)(1-\beta)(n \nu-\alpha)}{n+1-\beta},
\end{gathered}
$$

then $f \in \mathcal{S}_{1}(\beta) \subset \mathcal{S}^{*}(\beta)$. Equality is attained for $f(z)=$ $z+(1-\beta) z^{n+1} /(n+1-\beta)$.

Proof. Let

$$
p(z)=v z f^{\prime \prime}(z)-\alpha\left(f^{\prime}(z)-1\right) .
$$

A brief computation shows that

$$
p(z)+\mu z p^{\prime}(z)=\gamma z^{2} f^{\prime \prime \prime}(z)+\delta z f^{\prime \prime}(z)-\alpha\left(f^{\prime}(z)-1\right) .
$$

Hence, (15) can be written in the subordination form as

$$
p(z)+\mu z p^{\prime}(z) \prec \frac{(1+n \mu)(n+1)(1-\beta)(n \nu-\alpha)}{n+1-\beta} z .
$$

It follows from Lemma 1 that

$$
\begin{aligned}
p(z) & \prec \frac{1}{\mu n z^{1 / \mu n}} \int_{0}^{z} \frac{(1+\mu n)(n+1)(1-\beta)(n v-\alpha)}{n+1-\beta} t^{1 / \mu n} d t \\
& =\frac{(n+1)(1-\beta)(n v-\alpha)}{n+1-\beta} z,
\end{aligned}
$$

which implies

$$
\left|\nu z f^{\prime \prime}(z)-\alpha\left(f^{\prime}(z)-1\right)\right| \leq \frac{(n+1)(1-\beta)(n \nu-\alpha)}{n+1-\beta} .
$$

Hence $f \in \mathcal{S}_{1}(\beta) \subset \mathcal{S}^{*}(\beta)$ on using Lemma 3 .

For sharpness, it is evident that the function $f(z)=z+$ $(1-\beta) z^{n+1} /(n+1-\beta)$ satisfies

$$
\begin{gathered}
\left|\gamma z^{2} f^{\prime \prime \prime}(z)+\delta z f^{\prime \prime}(z)-\alpha\left(f^{\prime}(z)-1\right)\right| \\
=\frac{(1+n \mu)(n+1)(1-\beta)(n v-\alpha)}{n+1-\beta} .
\end{gathered}
$$


Thus,

$$
\left|\frac{z f^{\prime}(z)}{f(z)}-1\right|<1-\beta, \quad z \in U
$$

Theorem 7. Let $0<\alpha<n \mu, \delta>\alpha \geq \gamma \geq 0,0 \leq \beta<1$, and $g \in \mathscr{H}$. If

$$
|g(z)|<\frac{(1+\mu n)(n+1)(1-\beta)(n \nu-\alpha)}{n+1-\beta},
$$

where

$$
\nu-\alpha \mu=\delta-\gamma, \quad \gamma \mu=\gamma,
$$

then

$$
f(z)=z+\frac{z^{n+1}}{\gamma} \iiint_{0}^{1} g(r s t z) r^{n-1-\alpha / v} s^{n} t^{n-1+1 / \mu} d r d s d t
$$

satisfies $f \in \mathcal{S}_{1}(\beta) \subset \mathcal{S}^{*}(\beta)$.

Proof. Let $f \in \mathscr{A}_{n}$ satisfy

$$
\gamma z^{2} f^{\prime \prime \prime}(z)+\delta z f^{\prime \prime}(z)-\alpha\left(f^{\prime}(z)-1\right)=z^{n} g(z) .
$$

From Theorem 6, the solution of (26) belongs to the class $\mathcal{S}_{1}(\beta) \subset \mathcal{S}^{*}(\beta)$. Now (26) has the form

$$
p(z)+\mu z p^{\prime}(z)=z^{n} g(z),
$$

where

$$
p(z)=v z f^{\prime \prime}(z)-\alpha\left(f^{\prime}(z)-1\right) .
$$

Equation (27) has a solution

$$
\begin{aligned}
p(z) & =\frac{z^{-1 / \mu}}{\mu} \int_{0}^{z} g(\xi) \xi^{n-1+1 / \mu} d \xi \\
& =\frac{z^{n}}{\mu} \int_{0}^{1} g(t z) t^{n-1+1 / \mu} d t=z^{n} \phi(z),
\end{aligned}
$$

with

$$
\phi(z)=\frac{1}{\mu} \int_{0}^{1} g(t z) t^{n-1+1 / \mu} d t .
$$

Note that the function $f$ in Lemma 4 satisfies $\delta z f^{\prime \prime}(z)-$ $\alpha\left(f^{\prime}(z)-1\right)=z^{n} g(z)$. Thus replacing the appropriate parameters in the equation

$$
v z f^{\prime \prime}(z)-\alpha\left(f^{\prime}(z)-1\right)=z^{n} \phi(z)
$$

yields a solution

$$
\begin{aligned}
f(z) & =z+\frac{z^{n+1}}{v} \iint_{0}^{1} \phi(r s z) r^{n-1-\alpha / v} s^{n} d r d s \\
& =z+\frac{z^{n+1}}{\gamma} \iiint_{0}^{1} g(r s t z) r^{n-1-\alpha / v} s^{n} t^{n-1+1 / \mu} d r d s d t .
\end{aligned}
$$

This completes the proof.
The next result provides a sufficient condition for starlikeness of order $\beta$ involving a second-order differential inequality.

Lemma 8. Let $f \in \mathscr{A}_{n}$, and $0 \leq \alpha<\delta$ with $0 \leq \beta<1$. If

$$
\left|\delta z f^{\prime \prime}(z)-\alpha\left(f^{\prime}(z)-\frac{f(z)}{z}\right)\right|<\frac{n(1-\beta)(\delta(n+1)-\alpha)}{n+1-\beta},
$$

then $f \in \mathcal{S}_{1}(\beta) \subset \mathcal{S}^{*}(\beta)$ with an extremal function $f(z)=$ $z+(1-\beta) z^{n+1} /(n+1-\beta)$.

Proof. Inequality (33) can be expressed in the subordination form

$$
\begin{aligned}
\delta z f^{\prime \prime}(z)-\alpha\left(f^{\prime}(z)-\frac{f(z)}{z}\right) & \\
& \prec \frac{n(1-\beta)(\delta(n+1)-\alpha)}{n+1-\beta} z, \quad z \in U .
\end{aligned}
$$

Writing

$$
P(z)=f^{\prime}(z)-\frac{f(z)}{z}=P_{n} z^{n}+\cdots,
$$

it follows that

$$
\begin{aligned}
\delta z P^{\prime}(z)+(\delta-\alpha) P(z) & =\delta z f^{\prime \prime}(z)-\alpha\left(f^{\prime}(z)-\frac{f(z)}{z}\right) \\
& \prec \frac{n(1-\beta)(\delta(n+1)-\alpha)}{n+1-\beta} z .
\end{aligned}
$$

Now Lemma 1 with $\gamma=1-\alpha / \delta$ yields

$$
\begin{aligned}
P(z) & \prec \frac{\gamma}{n z^{\gamma / n}} \int_{0}^{z}\left(\frac{n(1-\beta)(\delta(n+1)-\alpha)}{(\delta-\alpha)(n+1-\beta)} t\right) t^{\gamma / n-1} d t \\
& =\frac{n(1-\beta)}{n+1-\beta} z,
\end{aligned}
$$

which implies

$$
\left|f^{\prime}(z)-\frac{f(z)}{z}\right|<\frac{n(1-\beta)}{n+1-\beta}
$$

Let

$$
p(z)=\frac{f(z)}{z}=1+p_{n} z^{n}+\cdots
$$

Since

$$
z p^{\prime}(z)=f^{\prime}(z)-\frac{f(z)}{z} \prec \frac{n(1-\beta)}{n+1-\beta} z
$$

an application of Lemma 2 shows that

$$
p(z) \prec 1+\frac{1}{n} \int_{0}^{z}\left(\frac{n(1-\beta)}{n+1-\beta} t\right) \frac{d t}{t}=1+\frac{(1-\beta)}{n+1-\beta} z .
$$


Therefore,

$$
|p(z)|=\left|\frac{f(z)}{z}\right|>1-\frac{1-\beta}{n+1-\beta}=\frac{n}{n+1-\beta} .
$$

Combining (38) and (42) yields

$$
\begin{aligned}
& \frac{n}{n+1-\beta}\left|\frac{z f^{\prime}(z)}{f(z)}-1\right| \\
& =\frac{n}{n+1-\beta}\left|\frac{z}{f(z)}\right|\left|f^{\prime}(z)-\frac{f(z)}{z}\right| \\
& \quad<\frac{n(1-\beta)}{n+1-\beta},
\end{aligned}
$$

which means $\left|z f^{\prime}(z) / f(z)-1\right|<1-\beta$, whence $f \in \mathcal{S}_{1}(\beta) \subset$ $\mathcal{S}^{*}(\beta)$.

Remark 9. For $\delta=1$ and $\beta=0$, Lemma 8 reduces to [12, Lemma 2.2].

The following result gives starlikeness for a function given by a double integral operator associated with Lemma 4 . The proof is analogous to Theorem 2.2 of [12] and is omitted.

Lemma 10. Let $0 \leq \alpha<n \delta, \delta>\alpha, 0 \leq \beta<1$, and $g \in \mathscr{H}$. If

$$
|g(z)|<\frac{n(1-\beta)(\delta(n+1)-\alpha)}{n+1-\beta}
$$

then

$$
f(z)=z+\frac{z^{n+1}}{\delta} \iint_{0}^{1} g(r s z) r^{(n \delta-\alpha) / \delta} s^{n-1} d r d s
$$

satisfies $f \in \mathcal{S}_{1}(\beta) \subset \mathcal{S}^{*}(\beta)$.

An application of Lemma 8 yields the following sufficient condition for starlikeness in terms of a third-order differential inequality.

Theorem 11. Let $f \in \mathscr{A}_{n}, 0 \leq \alpha<(1-\mu)(n+1) \nu, \alpha \geq \gamma \geq 0$, $\delta>\gamma+\alpha$, and $0 \leq \beta<1$. Further let

$$
\nu-\frac{\alpha \mu}{1-\mu}=\delta-\gamma, \quad \nu \mu=\gamma
$$

If

$$
\begin{gathered}
\left|\gamma z^{2} f^{\prime \prime \prime}(z)+\delta z f^{\prime \prime}(z)-\alpha\left(f^{\prime}(z)-\frac{f(z)}{z}\right)\right| \\
<\frac{n[(n+1)(\nu-\gamma)-\alpha](1-\beta)(1+n \mu)}{(n+1-\beta)(1-\mu)},
\end{gathered}
$$

then $f \in \mathcal{S}_{1}(\beta) \subset \mathcal{S}^{*}(\beta)$. Equality is attained for $f(z)=$ $z+(1-\beta) z^{n+1} /(n+1-\beta)$.
Proof. Proceeding similarly as in the proof of Lemma 8, inequality (47) can be written as

$$
\begin{gathered}
\gamma z^{2} f^{\prime \prime \prime}(z)+\delta z f^{\prime \prime}(z)-\alpha\left(f^{\prime}(z)-\frac{f(z)}{z}\right) \\
\prec \frac{n[(n+1)(\nu-\gamma)-\alpha](1-\beta)(1+n \mu)}{(n+1-\beta)(1-\mu)} z .
\end{gathered}
$$

Let

$$
p(z)=v z f^{\prime \prime}(z)-\frac{\alpha}{1-\mu}\left(f^{\prime}(z)-\frac{f(z)}{z}\right) .
$$

Then a computation yields

$$
\begin{aligned}
p^{\prime}(z)= & \nu z f^{\prime \prime \prime}(z)+\nu f^{\prime \prime}(z)-\frac{\alpha}{1-\mu} f^{\prime \prime}(z) \\
& +\frac{\alpha}{1-\mu}\left(\frac{f^{\prime}(z)}{z}-\frac{f(z)}{z^{2}}\right),
\end{aligned}
$$

so that

$$
\begin{aligned}
p(z)+\mu z p^{\prime}(z)= & \gamma z^{2} f^{\prime \prime \prime}(z)+\delta z f^{\prime \prime}(z) \\
& -\alpha\left(f^{\prime}(z)-\frac{f(z)}{z}\right)
\end{aligned}
$$

Hence

$$
\begin{aligned}
p(z) & +\mu z p^{\prime}(z) \\
& <\frac{n[(n+1)(\nu-\gamma)-\alpha](1-\beta)(1+n \mu)}{(n+1-\beta)(1-\mu)} z, \quad z \in U .
\end{aligned}
$$

Applying Lemma 1 yields

$$
\begin{aligned}
p(z) \prec \frac{1}{\mu n z^{1 / n \mu}} \int_{0}^{z}( & (n[(n+1)(\nu-\gamma)-\alpha] \\
& \times(1-\beta)(1+n \mu)) \\
& \left.\times((n+1-\beta)(1-\mu))^{-1}\right) t^{1 / n \mu} d t .
\end{aligned}
$$

This implies that

$$
\begin{gathered}
v z f^{\prime \prime}(z)-\frac{\alpha}{1-\mu}\left(f^{\prime}(z)-\frac{f(z)}{z}\right) \\
\prec \frac{n(1-\beta)[(n+1)(\nu-\gamma)-\alpha]}{(n+1-\beta)(1-\mu)} z,
\end{gathered}
$$

and thus

$$
\begin{gathered}
\left|\nu z f^{\prime \prime}(z)-\frac{\alpha}{1-\mu}\left(f^{\prime}(z)-\frac{f(z)}{z}\right)\right| \\
\leq \frac{n(1-\beta)[(n+1)(\nu-\gamma)-\alpha]}{(n+1-\beta)(1-\mu)} \\
\quad=\frac{n(1-\beta)[\nu(n+1)-\alpha /(1-\mu)]}{n+1-\beta},
\end{gathered}
$$


which, in comparison with Lemma 8, gives the required result.

Further the result is sharp for $f(z)=z+(1-\beta) z^{n+1} /(n+$ $1-\beta$ ) which satisfies

$$
\begin{gathered}
\left|\gamma z^{2} f^{\prime \prime \prime}(z)+\delta z f^{\prime \prime}(z)-\alpha\left(f^{\prime}(z)-\frac{f(z)}{z}\right)\right| \\
=\frac{n[(n+1)(\nu-\gamma)-\alpha](1-\beta)(1+n \mu)}{(n+1-\beta)(1-\mu)} .
\end{gathered}
$$

Remark 12. For $f \in \mathscr{A}_{n}$, the choice $\alpha=0=\gamma$ in Theorem 11 results in

$$
\left|\delta z f^{\prime \prime}(z)\right|<\frac{n[\delta(n+1)](1-\beta)}{n+1-\beta} \Longrightarrow f \in \mathcal{S}_{1}(\beta) \subset \mathcal{S}^{*}(\beta) .
$$

For $\delta=1$, this coincides with Lemma 8 at $\alpha=0$, which was also exhibited in [13, Corollary 2.4]. Further, for $n=1$, (57) gives

$$
\left|\delta z f^{\prime \prime}(z)\right|<\frac{2 \delta(1-\beta)}{2-\beta} \Longrightarrow f \in \mathcal{S}_{1}(\beta) \subset \mathcal{S}^{*}(\beta),
$$

which for $\delta=1$ and $\beta=0$ is the result given in $[8$, Theorem $1]$.

Corresponding to Theorem 11, a sufficient condition for starlikeness of order $\beta$ for functions defined by a triple integral operator is obtained in the following result.

Theorem 13. Let $0 \leq \alpha<(1-\mu)(n+1) \nu, \alpha \geq \gamma \geq 0, \delta>\gamma+\alpha$, $0 \leq \beta<1$, and $g \in \mathscr{H}$. Further let

$$
\nu-\frac{\alpha \mu}{1-\mu}=\delta-\gamma, \quad \nu \mu=\gamma .
$$

If

$$
|g(z)|<\frac{n[(n+1)(\nu-\gamma)-\alpha](1-\beta)(1+n \mu)}{(n+1-\beta)(1-\mu)},
$$

then

$$
\begin{gathered}
f(z)=z+\frac{z^{n+1}}{\gamma} \iiint_{0}^{1} g(r s t z) r^{(1 / v)[n v-\alpha /(1-\mu)]} \\
\times s^{n} t^{n-1+1 / \mu} d r d s d t
\end{gathered}
$$

satisfies $f \in \mathcal{S}_{1}(\beta) \subset \mathcal{S}^{*}(\beta)$.

Proof. Let $f \in \mathscr{A}_{n}$ satisfy

$$
\gamma z^{2} f^{\prime \prime \prime}(z)+\delta z f^{\prime \prime}(z)-\alpha\left(f^{\prime}(z)-\frac{f(z)}{z}\right)=z^{n} g(z) .
$$

From Theorem 11, we find that the solution of (62) lies in $\mathcal{S}^{*}(\beta)$. Now (62) becomes

$$
p(z)+\mu z p^{\prime}(z)=z^{n} g(z),
$$

where

$$
p(z)=v z f^{\prime \prime}(z)-\frac{\alpha}{1-\mu}\left(f^{\prime}(z)-\frac{f(z)}{z}\right) .
$$

Equation (63) has a solution

$$
\begin{aligned}
p(z) & =\frac{z^{-1 / \mu}}{\mu} \int_{0}^{z} g(\xi) \xi^{n+1 / \mu-1} d \xi \\
& =\frac{z^{n}}{\mu} \int_{0}^{1} g(t z) t^{n+1 / \mu-1} d t=z^{n} \phi(z),
\end{aligned}
$$

with

$$
\phi(z)=\frac{1}{\mu} \int_{0}^{1} g(t z) t^{n+1 / \mu-1} d t .
$$

In view of Lemma 10, the equation

$$
v z f^{\prime \prime}(z)-\frac{\alpha}{1-\mu}\left(f^{\prime}(z)-\frac{f(z)}{z}\right)=z^{n} \phi(z)
$$

has a solution

$$
\begin{gathered}
f(z)=z+\frac{z^{n+1}}{\nu} \iint_{0}^{1} \phi(r s z) r^{(1 / v)[n v-\alpha /(1-\mu)]} s^{n-1} d r d s \\
=z+\frac{z^{n+1}}{\mu \nu} \iiint_{0}^{1} g(r s t z) r^{(1 / v)[n v-\alpha /(1-\mu)]} \\
\quad \times s^{n-1} t^{n-1+1 / \mu} d r d s d t \\
=z+\frac{z^{n+1}}{\gamma} \iiint_{0}^{1} g(r s t z) r^{(1 / v)[n v-\alpha /(1-\mu)]} \\
\times s^{n-1} t^{n-1+1 / \mu} d r d s d t .
\end{gathered}
$$

This completes the proof.

\section{Conflict of Interests}

The authors declare that there is no conflict of interests regarding the publication of this paper.

\section{Acknowledgments}

The work presented here was supported in part by a research university grant from Universiti Sains Malaysia. The authors are thankful to the referees for their suggestions that helped improve the clarity of this paper.

\section{References}

[1] H. Silverman, "Univalent functions with negative coefficients," Proceedings of the American Mathematical Society, vol. 51, pp. 109-116, 1975. 
[2] N. Yagmur and H. Orhan, "Starlikeness and convexity of generalized Struve functions," Abstract and Applied Analysis, vol. 2013, Article ID 954513, 6 pages, 2013.

[3] R. M. Ali, "On a subclass of starlike functions," The Rocky Mountain Journal of Mathematics, vol. 24, no. 2, pp. 447-451, 1994.

[4] R. M. Ali and V. Singh, "Convexity and starlikeness of functions defined by a class of integral operators," Complex Variables: Theory and Application, vol. 26, no. 4, pp. 299-309, 1995.

[5] R. M. Ali, A. O. Badghaish, V. Ravichandran, and A. Swaminathan, "Starlikeness of integral transforms and duality," Journal of Mathematical Analysis and Applications, vol. 385, no. 2, pp. 808-822, 2012.

[6] R. M. Ali, M. M. Nargesi, and V. Ravichandran, "Convexity of integral transforms and duality," Complex Variables and Elliptic Equations, vol. 58, no. 11, pp. 1569-1590, 2013.

[7] R. Fournier and P. T. Mocanu, "Differential inequalities and starlikeness," Complex Variables: Theory and Application, vol. 48, no. 4, pp. 283-292, 2003.

[8] M. Obradović, "Simple sufficient conditions for univalence," Matematichki Vesnik, vol. 49, no. 3-4, pp. 241-244, 1997.

[9] R. Omar, S. A. Halim, and R. W. Ibrahim, "Differential subordination properties of certain analytic functions," International Journal of Mathematics, vol. 24, no. 6, Article ID 1350044, 7 pages, 2013.

[10] R. Omar and S. A. Halim, "Differential subordination properties of Sokol-Stankiewiczstarlike functions," Kyungpook Mathematical Journal, vol. 53, no. 3, pp. 459-465, 2013.

[11] H. Shiraishi, K. Kuroki, and S. Owa, "Some classes of order for second-order $\alpha$ differential inequalities," Electronic Journal of Mathematical Analysis and Applications, vol. 1, no. 2, pp. 149155, 2013.

[12] S. S. Miller and P. T. Mocanu, "Double integral starlike operators," Integral Transforms and Special Functions, vol. 19, no. 7-8, pp. 591-597, 2008.

[13] K. Kuroki and S. Owa, "Double integral operators concerning starlike of order $\beta$," International Journal of Differential Equations, vol. 2009, Article ID 737129, 13 pages, 2009.

[14] R. M. Ali, S. K. Lee, K. G. Subramanian, and A. Swaminathan, "A third-order differential equation and starlikeness of a double integral operator," Abstract and Applied Analysis, vol. 2011, Article ID 901235, 10 pages, 2011.

[15] D. J. Hallenbeck and S. Ruscheweyh, "Subordination by convex functions," Proceedings of the American Mathematical Society, vol. 52, pp. 191-195, 1975.

[16] S. S. Miller and P. T. Mocanu, Differential Subordinations, Marcel Dekker, New York, NY, USA, 2000.

[17] T. J. Suffridge, "Some remarks on convex maps of the unit disk," Duke Mathematical Journal, vol. 37, pp. 775-777, 1970. 


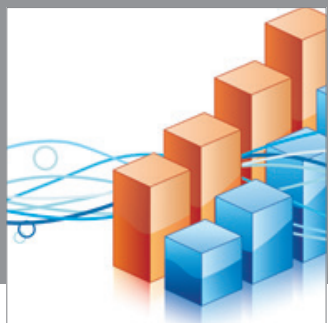

Advances in

Operations Research

mansans

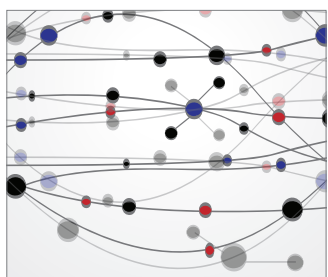

The Scientific World Journal
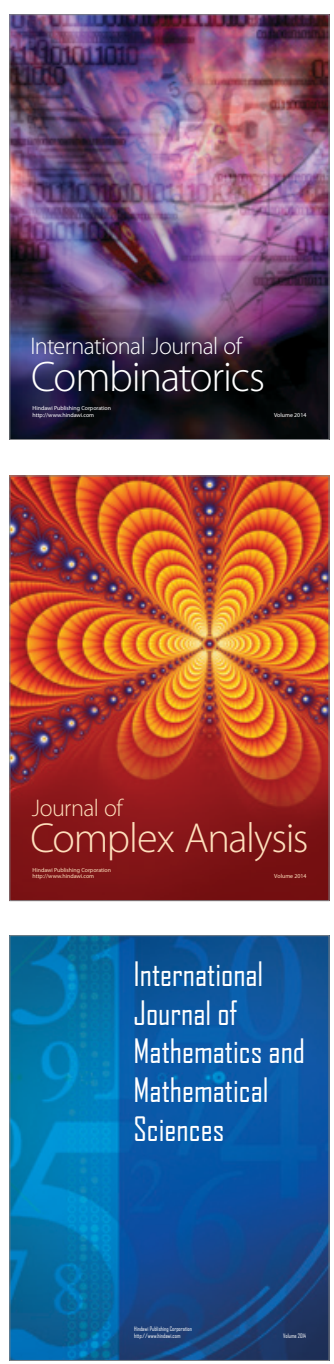
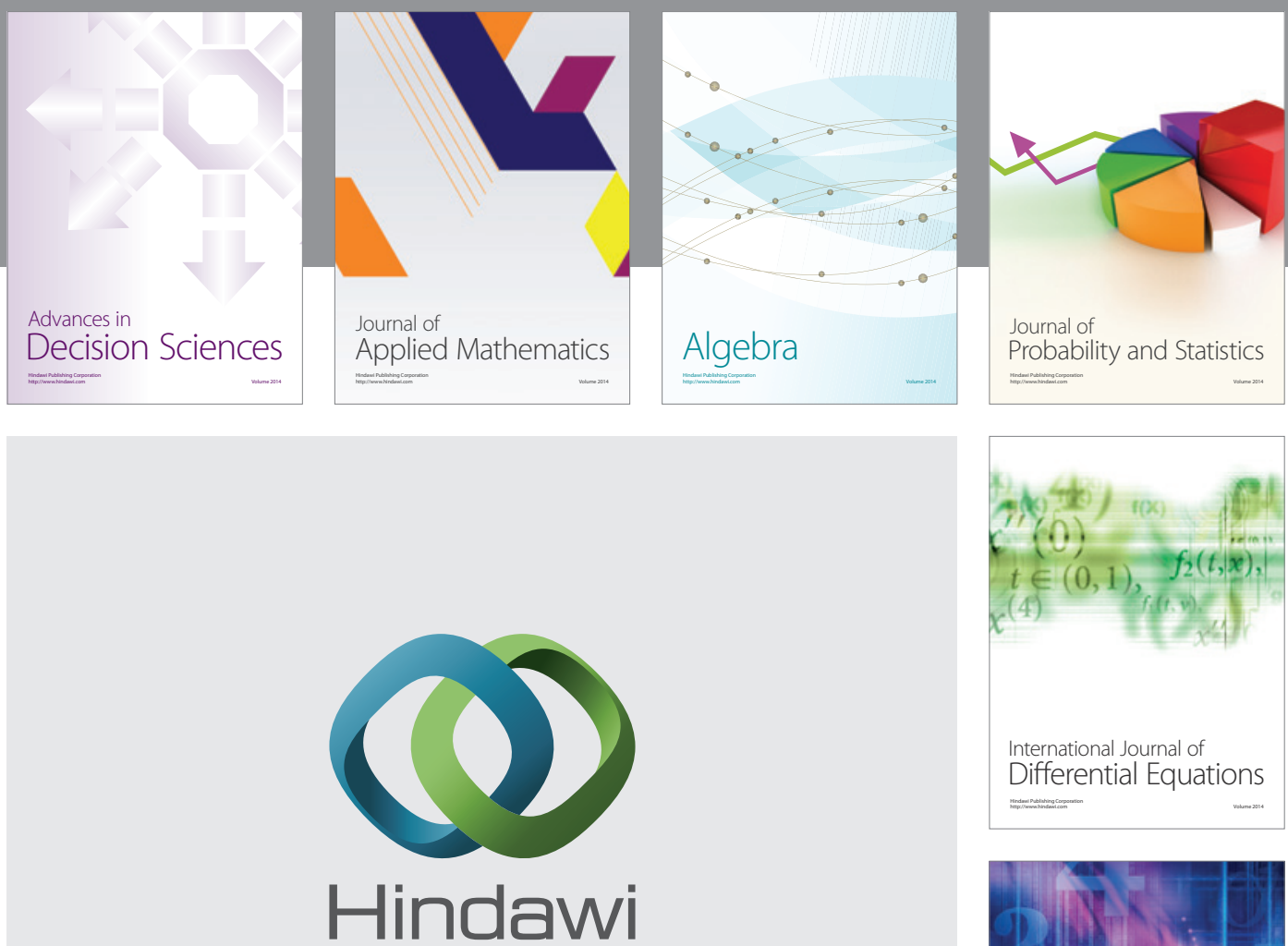

Submit your manuscripts at http://www.hindawi.com
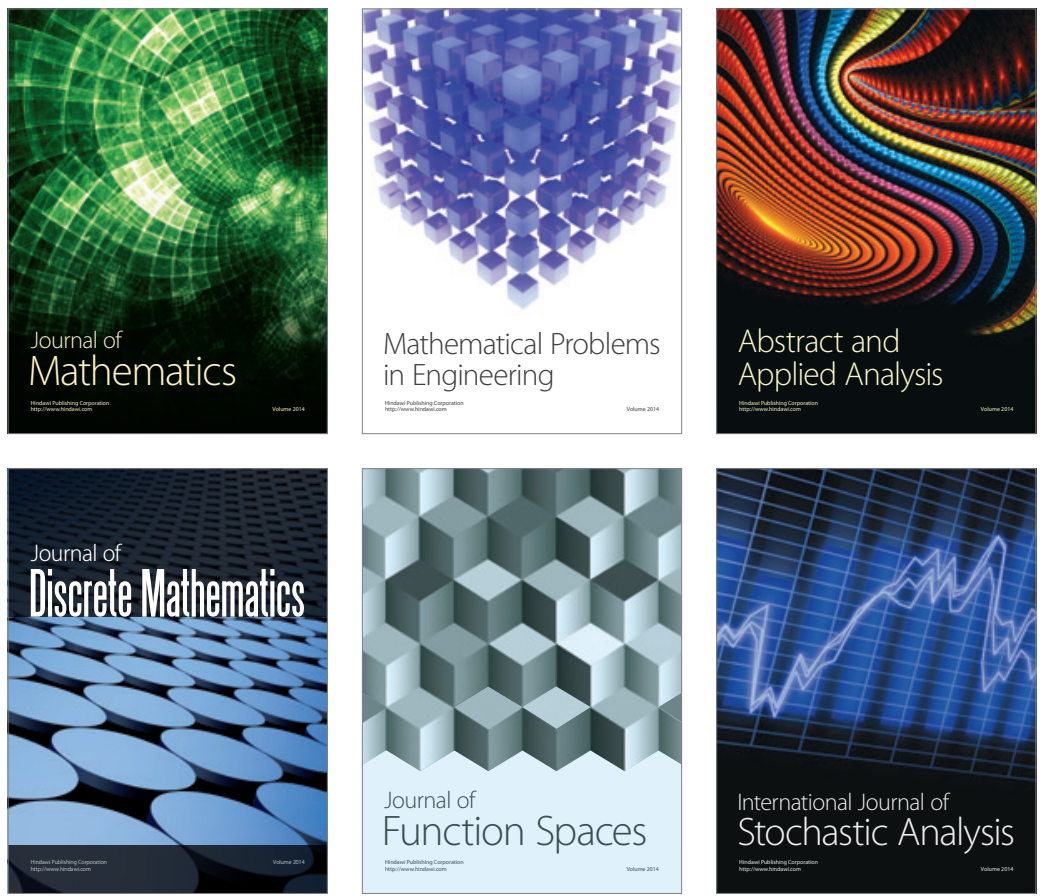

Journal of

Function Spaces

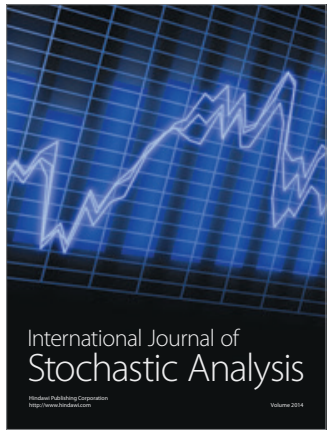

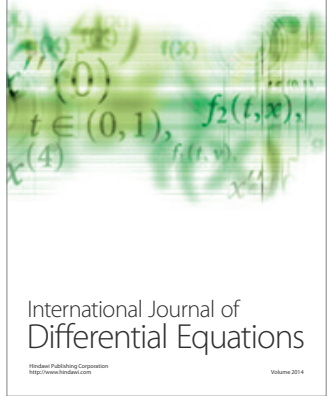
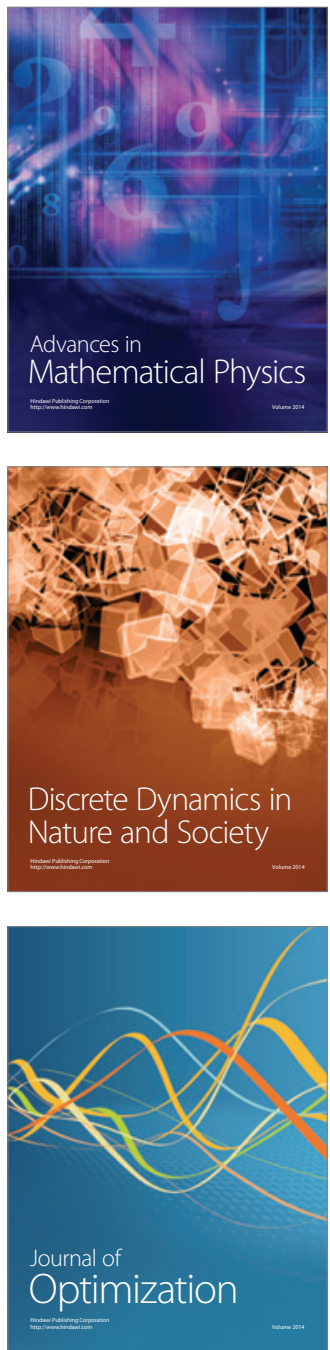